\title{
Procedural invariants of discovery process
}

\section{Georgy Morgunov}

Department of philosophy, Novosibirsk State Technical University, Russia

\author{
Email address: \\ coliseygm@mail.ru
}

\section{To cite this article:}

Georgy Morgunov. Procedural Invariants of Discovery Process. International Journal of Philosophy. Vol. 2, No. 2, 2014 , pp. $26-30$. doi: $10.11648 /$ j.ijp. 20140202.12

\begin{abstract}
The article reveals a synergistic approach to understanding of creativity. Possibility of applying Vernadsky's ideas to interpret creativity is shown with the role and importance of his mental component. Shown discussion about the creativity process and possible requirements for its successful start. Described noosphere force from creativity point of view.
\end{abstract}

Keywords: Discovery, Noosphere, Synergetics, Creativity

\section{Introduction}

Discovery is the process of identifying something new, that hasn't been known before. Although it is very important phenomena for humanity, because it allows to expand the general knowledge, it is not researched well enough. The main question that arises is what are necessary conditions and prerequisites for discovery to happen? The discussions and attempt to answer this question are made in the chapter 2 .

\section{Creativity}

An important step in clarifying the ontological content of creativity is a synergistic modeling of reality. Synergetics allows substantial progress in identifying procedural invariants of global innovation process.

The summary of synergetics achievements is as follows:

1. "The source of the order is disequilibrium. Disequilibrium is what generates "order out of the chaos", every birth of new (order) is preceded by non-equilibrium state of complex nonlinear open statistical system, where the emergence of a new is only possible.

2. Disequilibrium results in the system's bifurcation with the spontaneous nucleation of attractors stable non-equilibriums. Bifurcations are unpredictable because of their indeterminacy.

The appearance of attractors initiates system adaptation to the environment. These new attractors usually subordinate non-equilibrium statistical system and the whole system is rebuilt to a new steady state. Thus "dissipative" structure is formed, that is the order of the "new", not previously existing type.

On the other hand, new dissipative structure in the strong non-equilibrium state is transformed into a system with a different, higher index of order. As a result, the algorithm of any discovery of "new", according to the synergy, is as follows:

disequilibrium - bifurcation - adaptation.

The authors of the synergetics describe procedural specifics of becoming as "... Close to origin point of instability one can distinguish between stable and unstable collective moves (modes). Stable modes can adjust to unstable and thus can be removed. In general this leads to a tremendous reduction in the degrees of freedom. Remaining unstable modes serve as parameters of order that determine the macroscopic behavior of the system." [3] Prigogine and Stengers [1] are also close to this understanding: "When the thermodynamic forces, which act on the system, become sufficiently large and force it to leave the linear region, guarantee of the stability of steady state or its independence from fluctuations would be reckless. Outside the linear region stability is no longer a consequence of the general laws of physics. It is necessary to research in what way steady state reacts on different types of fluctuations generated by the system or the environment. In some cases, the analysis leads to the conclusion that steady state is unstable. In these situations certain fluctuations, instead of fading, amplify and obsess the entire system, forcing it to evolve to the new regime, which may be qualitatively different from the steady 
states, corresponding to the minimum entropy production". We specifically use the terminology of synergy in the methodology of innovation. This enables new, neutral terms to define general structure of increment regardless of its particular nature or environment.

Prigogine's discovery of general structure formation on the material of inanimate nature is of specific methodological significance. We observe rising of new scientific statistical concept of the world. Not all parts are clear, and a lot of ideas are needed to be developed, but the overall draft is already there. General and basic form of motion of substance is the thermal (statistical) one. Each phenomenon is probabilistic. In the world there is a statistical stability and equilibrium, but the leading role belongs to the instability and disequilibrium. All material systems contain subsystems that continually fluctuate. Sometimes separate fluctuation or combination of several ones may be so severe that previously existed organization does not hold and collapses. At this critical point (bifurcation point) is fundamentally impossible to predict the direction in which the development will occur. There are two ways - to the higher level of organization (creation of dissipative structures) or downshift to the old stationary or chaotic state. Every phenomenon that currently exists in the universe was arisen as a result of disequilibrium through bifurcations and represents an attractor of corresponding open nonlinear system. However, each existing attractor will be destroyed with the same reason and in the same manner as he has replaced the previous steady state.

According to the ideas of synergetic world we can observe creativity as global cosmic process, performed out in the spatial and temporal boundaries of the planet Earth.

Starting point for this world is the statistical organisation - life of billions of people with their own wills, actions and desires - that is the life of complicated, nonlinear, open statistical system, whose name - society. At least, there are two modes of existence of society balance and imbalance. Imbalance is a driver for creativity. It results in a bifurcation of system with spontaneously appearing attractors - science, art, government, empires and civilizations. Within each of the attractors own stable disequilibriums, with certain periods of existence, are growing (within subsystems). Attractors adapt the system subsystems and by themselves, through disequilibrium, disappear into formation of new attractors, whose fate is to be the basis for other development.

The picture of creativity shown above, without additional specificity, is of allegorical, metaphysical and, in the best case, analogy character. Used terms like "bifurcation", "disequilibrium", "attractors", "open system", etc., are precisely defined in relation to dynamic systems, in the case of non-stationarity and evolutionarity they are described by relevant dynamical equations, but in relation to creativity are not sufficiently developed. However, nowadays it should be done,
In his works Vernadsky has accumulated sufficient methodological material for science analysis of creativity. Quantitative characteristics of living substance Vernadsky expresses not in biological (species, populations, families, genera), but in geochemical units (chemical formula, weight, energy). As a result, large migrations of organisms (for example, birds, insects' swarms, fish schools) are characterized in the same way as moving rocks. Thus joint starting point for geological and biological phenomena is formed, terms "weight", "power", "energy" are becoming possible to be used in the physical sense as the basic parameters of evolution, what allows us to use the apparatus of mathematical physics in the study of living systems. The same is to be done in the field of creativity, in the analysis of psychic phenomena. It is the time to use language of "force", "energy", "mass", "divergence", "rotation" in this area and refuse (under the proposed analysis) from the terms of the humanities, which complicate the mathematical study of creativity. It is time to fill the terminology of humanities sciences with "power" and "energy" content to consider feelings, mind and imagination as some characteristics of specific power and energy. Then there will be the basis for applying mathematical tools of the natural sciences in the field of mental and social phenomena and the ability to refine the concept and conceptual analysis, which dominate in the analysis of creativity, expressed in terms of formulas and equations in relation to the force, mass, energy and creative flow of information. Until this is done, we will work with an accuracy of categorical analysis of creativity, which is usually much lower than the corresponding ones, based on systems of equations.

Blyumenfeld [5] has calculated that spontaneous occurrence of even a single protein molecule based on physical force is beyond of the reasonable possibility. The probability of random occurrence of one DNA molecule for the whole history of the Earth at a synthesis frequency 100 million variants per second is the meaningless value of 10800 years. Thus it is easy to draw a conclusion about the existence of the vital forces, different from physical ones and responsible for those specific phenomena that constitute evolution.

Spontaneous emergence of creative phenomena lies beyond reasonable possibility within the vital (biological) forces. Assume that there are hundred chimpanzees sitting at typewriters and each of them randomly prints letters on clean sheets of paper. Other chimpanzees snatching each printed sheet and pass it to the center, where other chimpanzees brochures books with them. There is a chance that one of the books "produced" in this way will be a full copy of "War and Peace" by Leo Tolstoy [6]. However, the probability of this event is extremely low and is comparable to the probability that the table, under the influence of Brownian motion, will go up. There is also a possibility that chimpanzees in a larger, but finite time could "produce" all literary masterpieces. But this 
probability is so small that it is pointless to think about creativity convergence to the physical and vital (biological) forces.

These examples allow us to make a methodological conclusion about existence of "special forces" (let's call them, in the same way as Vernadsky, the "noospheric"), responsible for the occurrence of probable, but impossible within the physical and biological worlds, phenomena, constituting creativity. We need to admit - there are exist probable, but impossible for some forces, events. There is a difference between probability and possibility: events can be probable, but impossible under varying nature. In addition to differentiation between the equilibrium (where the entropy production, as well as flows and forces are zero), weak-equilibrium (where the thermodynamic forces and flows depend linearly on the forces) and strong-equilibrium processes (where flows are nonlinear, and the entropy increase goes into dissipative structures), there is another significant distinction of probabilistic processes: all the phenomena are divided into probable, improbable, feasible and probable, but impossible in an inanimate matter. Synergetics examined only first two kinds of processes. Therefore, the model of synergetic analysis of innovation processes, reproduced above in relation to creativity, should be updated. Synergetics, in the form it was explained by Haken and Prigogine, is insufficient for a full analysis of creativity as a form of innovation processes. It does not overcome the gap between the animate and inanimate, on the one hand, and society - on the other, because it does not cover all variety of forces that make up the existing world. The probable, but not implemented by inanimate nature, area is not covered by synergy. But this area exists. Facts have proven that in the statistical world, in addition to the entropy barrier, which prohibits transitions from the future into the present and the past, there are still a number of barriers, which makes the surrounding world strange and specific. Indeed, the process of emergence of animate from inanimate via reproduction looks naturally simple and clear in the current state of science. But it is unclear why under conditions of spreading of animate on Earth there are no observed processes of direct emergence of animate from inanimate? Why with availability of ontogenesis and phylogenesis the orthogenesis - the emergence of human from primate - is not possible? There are other known restricted areas. Quote: "But, of course, proved the presence of such, if it is possible to say so, "suggestions" in the genetic language that can not be realized, such sequences in the genetic material, which theoretically should exist, but not opened by nature. All this tells us that the flow of mutations is not broken into separate random streams, but is strictly ordered in channels".

Similarly, today the production of car seems natural routine for developed countries. However, the appearance of the car in wildlife, out of society is probable, but not possible. It seems that universe, will not find a teaspoon, without the humanity. In this regard, it is logical to assume another forbidding principle in the probabilistic inanimate world, which prohibits spontaneous occurrence of such events as "War and Peace" of Tolstoy, aircraft, architecture of Kremlin, etc., that is, excluding the occurrence of what is called the result of creativity.

But the Kremlin architecture exists, "War and Peace" is printed in millions of copies, planes do fly, etc., so this means that the process of overcoming the barrier, unreachable for inanimate forces, is successfully implemented. The only thing left to understand is how this happens, whereby and why?

There are a lot of rumors about secret mystery of creativity and it is usually associated with some intimate psychological side of creativity. It seems that the real secret of creativity is why probable, but impossible for non-stationarity and evolutionarity, events happen, how to overcome the barrier of probable, but impossible for inanimate matter. Author supposes that there is another possible explanation - the process of overcoming the mentioned barrier, i.e. creativity, is "happens" according to the pattern "disequilibrium - bifurcation - adaptation" and each of its elements is carried out not only by the animate and inanimate nature, but also by the means of specific, so-called "noosphere" force, as presence of probable, but impossible for the usual statistical processes of inanimate in processes of speciation, proves the existence of special "vital forces" inside animate, the same as presence of probable, but impossible for the events of inanimate nature of the third world, proves the existence of a mechanism for creating and changing the third nature, based on the special forces. This thesis seems so to be of such importance that it is reasonable to analyze the meaning of noosphere force.

Contrary to the myth of freedom as a perceived necessity, life experience shows that man does not live in a strictly deterministic world, where everything is subordinated to the needs and patterns, in a world that is "soft", "flexible", statistical and probabilistic. Creator sees this world as partially ordered set of processes with local space-temporal structures, kaleidoscopic combination of appearing and disappearing stable formations. Mosaic, sustainability contours are present, but emerge as addition of physical, chemical, biological and other forces. Noosphere force is among them.

Origin point, control and regulating factor for noosphere force, is energy, in the psychological literature known as "the will and desire." It is accepted that people's will and desire are considered as an ideal combination, which is in this case, is unclear how to be dealt with, how to relate to the material world, except the reflection. At the same time will and desire - are the real physiological processes capable to organize (this is the essence of the will and desire) other physiological (and through them mechanical, physical and biochemical) processes of the human body with respect to structure and goals, contained in will and desire of people. Let's introduce notation for 
people's will and desire, as well as for similar psychic phenomena, the term "psychotropic force" [9]. Accordingly, next terms are introduced: "psychotropic energy", "psychotropic momentum", "spread speed" and others, which describe the physical aspects of any force. Psychotropic effect on the outer world and on the structure of the human body has every person. There are known methods of objective registering of psychotropic effects (in particular, imagination) by using a photo- and magneto- tape recorder technology. Psychotropic effects are relatively small: in photographing hallucinations used tapes with sensitivity of about 500 units. Psychics' psychotropic effects are much stronger. The small size of the energy power value of psychotropic effects (strong-will, in particular) is obvious; will is not enough to heat the water, but it's enough to set in motion the muscular structure of the human body, and through the organs of the body be involved in change of surrounding world, in accordance with the will and desire of person. Thus, psychotropic effects in the structure of the noosphere, are the starting processes of motion, like an explosion of ignition device, which activates the explosion of the main unit, with higher power than ignition device itself. Psychotropic effect is the controlling process, mobilizing all existing natural forms of energy in the form of motion of the noosphere. It does not satisfy the principle of "correctness" [10].

Second constituent elements of noosphere force, directly connected to psychotropic processes, are physiological processes in the muscles of the human body, partially controlled by psychotropic movement. The energy of these processes is much higher than power of psychotropic sourcing. Psychotropic energy of consciousness, together with physiological energy of the body gives opportunity to human to confront the nature as a mechanical force, and the ability to give a material nature to a form suitable for his life, by moving legs, head and fingers.

Social organization of people even more strengthens psychotropic effects, already powered by physiological processes organized in the human body. Here social energy, which, as we said, is strengthened by psychotropic energy, grows to geological and, sometimes, cosmic impacts. Emerged by multiple increased psychotropic impact through a chain of "physiology - society technology" natural impulse of energy, which is the will and desire of people, is the material unit, which interaction with natural forces, existing and acting objectively, define images of the Earth as a whole and in different parts, which had and currently have Earth during the evolution and existence of mankind.

Engels said that the summation of humans' wills in the result of the historical process, from the perspective of content, does not match with the content of initial wills and desires of people, resulting in the fact that historic acts are objective, independently of the will to desire. But it is not always true. In organized communities of authoritarian type, usually there is an opposite situation the content of the historical actions coincides with the initial content of the wills and desires of some people at power. In communities working as totalitarian systems, the will of one man determines the content of the historical actions of millions of people.

It is also assumed that a person adapts to objectively existing reality, he only succeeds when his actions "fit" it. Otherwise person fails. But this is more complicated. Noospheric impact acts as summation of natural forces, which define the picture of the world, as an objective factor and is a part of system of equations, in the same way as other forces of space, physical and planetary origin, describing the behavior of some part of the Earth, solar system or cosmic process.

For the phenomena of planetary scale, "contribution" of noosphere action now is the decisive - Earth image is defined by the will and desire of people. At the level of the solar system, noosphere influence on nature is less than the forces of the solar level. Cosmic impact of noosphere force is miserable. Noospheric force does not adapt to the other natural forces. In some cases when the values of its energy are small, in comparison with other natural forces, it can be neglected. In other cases, on the contrary, natural forces can be neglected. In some cases, the combination of the natural and noosphere forces, through functional natural relationships, which are expressed in systems of equations, determine the actual situation of some part of the Universe.

\section{Conclusion}

Considering everything said above, we can interpret the famous thesis "consciousness creates the world around us" in a different way: projects, programs, that are emerged in their own immanent way (via reflection, which is not enough) are transformed into the form of wills and desires of individuals and groups, amplified by biological means, organized into our bodies, and through them reach the communities and machines that multiply the power of consciousness to a geological level.

\section{References}

[1] Prigogine I., Stengers I. - Order out of chaos. - M., 1986.

[2] Haken H. Synergetics. - M ., 1980. - p .379.

[3] Blyumeifeld LA Problems of Biological Physics - L., 1997. P.16.

[4] Gaynullina L., Agapov O., Synergetic anthropology as new humanitarian paradigm// Question of philosophy, 2010, №5.

[5] Balaban V., Synergy: origins and manifestations. Vladivostok: TGEU, 2008.

[6] Alekseev V., Formation of mankind. - M., 1984. - P. 
[7] Burgis E. Intensivkurs: Allgemeine und spezielle Pharmakologie. - 2., aktualisierte Auflage. - München -Jena: Urban \& Fischer, 2002. - S. 10; Kütter T. Allgemeine Pharmakologie und Toxicologie. - 18., überarbeitete Auflage. - München - Jena: Urban \&Fischer, 2002.
[8] Solovyov V., Social systems theory -Novosibirsk: SBoRPA, 2005. 